\title{
Effect of chondrocyte mitochondrial dysfunction on cartilage degeneration: A possible pathway for osteoarthritis pathology at the subcellular level
}

\author{
HENG LIU*, ZHUOYANG LI*, YONGPING CAO, YUNPENG CUI, \\ XIN YANG, ZHICHAO MENG and RUI WANG \\ Department of Orthopedics, Peking University First Hospital, Beijing 100034, P.R. China
}

Received October 4, 2018; Accepted April 4, 2019

DOI: $10.3892 / \mathrm{mmr} .2019 .10559$

\begin{abstract}
Previous studies identified that chondrocyte apoptosis serves an important role in osteoarthritis (OA). However, the mechanisms of cartilage degeneration induced by apoptosis remain unclear. The present study investigated the role of mitochondrial dysfunction in OA pathology. A total of 30 cartilage samples presenting an Outerbridge score ranging between 0 and III were collected during total knee arthroplasty. Half of the samples were embedded for observation by transmission electron microscopy. The remaining samples were digested, and chondrocytes were isolated from normal and OA tissues. Subsequently, the enzymatic activity of factors of the mitochondrial respiratory chain (MRC), and mitochondrial membrane potential $(\Delta \psi \mathrm{m})$, were quantified. Furthermore, chondrocytes were treated with rotenone (Ro), a specific inhibitor of the MRC, and curcumin (Cur), a mitochondrial protective agent, with the aim of analyzing the relationship between mitochondrial dysfunction and chondrocyte apoptosis. The mitochondria of OA chondrocytes showed apoptosis-associated morphological alterations compared with normal cells. The $\Delta \psi \mathrm{m}$ and the activity of MRC enzymes were decreased in OA chondrocytes. Moreover, compared with normal chondrocytes, treatment with Ro was able to induce morphological changes reminiscent of the phenotype observed in OA chondrocytes. Additionally, Ro inhibited cellular proliferation, increased the apoptotic rate, and decreased the $\Delta \psi \mathrm{m}$ and the secretion of type II collagen. Furthermore, Cur could partly reverse the effects caused by treatment with Ro. The present data suggested that mitochondrial function was
\end{abstract}

Correspondence to: Dr Yongping Cao, Department of Orthopedics, Peking University First Hospital, 8 Xishi Ku Street, Beijing 100034, P.R. China

E-mail: freehorse6666@163.com

\section{*Contributed equally}

Key words: osteoarthritis, chondrocyte, mitochondrial dysfunction, rotenone, curcumin impaired in OA chondrocytes, resulting in an increased chondrocyte apoptosis and decreased type II collagen secretion. In addition, treatment with Cur protected the mitochondrial function and prevented cartilage degeneration. Collectively, the present results suggested that mitochondrial dysfunction may aggravate cartilage degeneration in the pathogenesis of OA.

\section{Introduction}

The critical role of chondrocytes in the etiology of osteoarthritis (OA) was previously described (1). However, the molecular mechanism underlying OA remains poorly understood. Chondrocytes are the only functional cells in cartilage, and they regulate the synthesis, deposition and modification of the extracellular matrix (ECM). Normal human articular cartilage is primarily composed of ECM with chondrocytes embedded in it (2). In the past, cartilage was considered to be a tissue with limited blood supply and low oxygen tension that relied on the glycolytic pathway, rather than mitochondria, for energy production (3). However, previous studies demonstrated that oxygen tension in cartilage is $5-10 \%$ at the surface and $<1 \%$ in deeper layers of the tissue $(4,5)$. Notably, the cartilaginous tissues facing the synovial fluid are exposed to relatively normal oxygen pressure $(4,5)$. Therefore, cartilage can present aerobic respiration via the mitochondrial pathway and the tricarboxylic acid (TCA) cycle (6).

A number of previous studies have demonstrated that chondrocytes are able to produce energy through aerobic metabolism (6-8). Chondrocytes express mitochondrial dehydrogenase, and isolated mitochondria from chondrocytes contain enzymes for oxidative phosphorylation and electron transfer (6-8). Additionally, mitochondria isolated from chondrocytes can use substrates of the TCA cycle to produce energy (6-8). Although aerobic respiration accounts for the degradation of only $20 \%$ of the carbohydrates in chondrocytes, the efficiency of oxidative phosphorylation in terms of ATP production is higher than that of anaerobic glycolysis (7). Therefore, mitochondrial aerobic respiration is one of the primary pathways used to produce energy in chondrocytes. Changes in chondrocyte function, including the ability to secrete type II collagen, and in chondrocyte apoptotic rates 
can directly affect the occurrence and development of articular cartilage degeneration in OA (9). However, whether mitochondrial dysfunction can affect chondrocyte function, leading to OA, remains unclear.

Enzyme complexes involved in the electron transport in the respiratory chain are present in the mitochondrial inner membrane (10). Oxidative phosphorylation and electron transport involve the enzyme complexes I-IV, and ATP is synthesized from ADP by ATP synthase, at the end of the electron transport chain (11). The electron transport chain generates a potential difference in the mitochondrial inner membrane, which is necessary for the synthesis of ATP (12). The mitochondrial membrane potential $(\Delta \psi \mathrm{m})$ plays an important role in maintaining mitochondrial membrane permeability and mitochondrial function (13). Therefore, the integrity of the mitochondrial respiratory chain (MRC) is important for the synthesis of ATP and for the maintenance of $\Delta \psi \mathrm{m}$, and defects in the respiratory chain or in the process of ATP synthesis may lead to mitochondrial dysfunction.

Rotenone (Ro) is a natural isoflavone produced by leguminous plants, and it is a specific inhibitor of the respiratory chain enzyme complex I (14). Various previous studies have used Ro to block the respiratory chain $(15,16)$. Curcumin (Cur) is an active pharmacological component extracted from plants in the genus Curcuma (17). Cur possesses strong anti-inflammatory and antioxidant effects that can effectively decrease the intracellular level of reactive oxygen species (ROS), stabilize the mitochondrial membrane and protect mitochondrial function $(18,19)$.

Therefore, the present study investigated the differences in mitochondrial morphology and function between OA and normal cartilage, with the aim of examining mitochondrial function in chondrocytes from OA cartilage. Furthermore, treatments with Ro and Cur were performed to affect mitochondrial function and to examine chondrocyte apoptosis and collagen secretion.

\section{Materials and methods}

Sample data. A total of 30 cartilage samples were collected from the femoral and tibial plateau of patients who underwent total knee arthroplasty due to osteoarthritis at The Peking University First Hospital between April and October 2016. Patients undergoing knee arthroplasty for other reasons were not included. In total, 7 male and 23 female patients (age $72 \pm 6$ years, age range 59-78) were enrolled in the present study. According to the Outerbridge grading system (20), the samples ranged from Outerbridge grade 0 to III. In total, 50\% of the samples were immersed in neutral formalin decalcification solution containing $10 \%$ EDTA at a volume ratio of 1:100. The decalcification solution was replaced every week. After the subchondral bone softened, the samples were able to be embedded in paraffin. The remaining samples were digested with the modified Klagsbrun method to harvest chondrocytes (21). Briefly, the cartilage was cut into small pieces $\left(1 \mathrm{~mm}^{3}\right)$ and digested with $0.25 \%$ trypsin (Nanjing KeyGen Biotech Co., Ltd.) solution for $30 \mathrm{~min}$. Then it was digested with sterile $0.2 \%$ collagenase (Nanjing KeyGen Biotech Co., Ltd.) for $5 \mathrm{~h}$. The resulting cell suspension was filtered twice, and isolated chondrocytes were seeded in ventilated $75 \mathrm{~cm}^{2}$ Costar polystyrene monolayer cell culture flasks (Corning, Inc.). The flasks were maintained at $37^{\circ} \mathrm{C}, 5 \% \mathrm{CO}_{2}$ and $100 \%$ humidity. The present study was approved by The Ethics Committee of Peking University First Hospital, and all participants provided informed consent.

Histological observation. The embedded samples were cut into $4 \mu \mathrm{m}$-thick paraffin sections. The overall chondrocyte morphology was evaluated by hematoxylin $\left(10 \mathrm{~min}, 37^{\circ} \mathrm{C}\right)$ and eosin $\left(3 \mathrm{~min}, 37^{\circ} \mathrm{C}\right)$ staining, the apoptosis of the chondrocytes was observed by TUNEL $\left(60 \mathrm{~min}, 37^{\circ} \mathrm{C}\right)$ staining (Roche Diagnostics) and light microscopy was used to image the samples (magnification, x100). Moreover, cartilage from the operation room was cut into $3 \times 5 \times 5 \mathrm{~mm}$ pieces. The specimen was quickly placed in 3\% glutaraldehyde solution for $24 \mathrm{~h}$ and $1 \%$ citric acid for $1 \mathrm{~h}$ at $4^{\circ} \mathrm{C}$. It was then dehydrated in an ascending gradient of ethanol and acetone (50, 70, 90 and 100\%). The tissue was embedded with Epon 812 resin overnight at $37^{\circ} \mathrm{C}$ and localized by semi-thin section (thickness, $0.5-2.0 \mu \mathrm{m}$ ). Ultra-thin sections (thickness, $<0.1 \mu \mathrm{m}$ ) were stained with uranium acetate and lead citrate. The sections were subjected to transmission electron microscopy (TEM).

Chondrocyte culture. Normal and OA chondrocytes were harvested from normal (Outerbridge grade 0) and OA (Outerbridge grade I-III) cartilage samples following digestion, according to the aforementioned procedure. Chondrocytes were cultured in DMEM (HyClone; GE Healthcare Life Sciences) supplemented with $10 \%$ FBS (HyClone; GE Healthcare Life Sciences) and $1 \%$ penicillin-streptomycin (Nanjing KeyGen Biotech Co., Ltd.). The cells were maintained in a humidified atmosphere containing $5 \% \mathrm{CO}_{2}$ and at $37^{\circ} \mathrm{C}$.

Mitochondrial function assessment in chondrocytes. Normal and OA chondrocytes were harvested as mentioned above to prepare a monoplast suspension of $>1 \times 10^{7}$ cells $/ \mathrm{ml}$. Mitochondria were extracted using a mitochondrial extraction kit (Nanjing KeyGen Biotech Co., Ltd.) according to the manufacturer's protocol, and the activities of the mitochondrial enzymes were calculated at $25^{\circ} \mathrm{C}$ using the initial velocity equation for pseudo-first-order reactions, as previously described $(5,22)$.

$\Delta \psi m$ measurement of chondrocytes. Normal and OA chondrocytes were harvested to prepare a monoplast suspension of $>1 \times 10^{5}$ cells $/ \mathrm{ml}$. Chondrocytes were stained with JC-1 using a $\Delta \psi \mathrm{m}$ assay kit (Sigma-Aldrich; Merck $\mathrm{KGaA}$ ) at $37^{\circ} \mathrm{C}$ for $20 \mathrm{~min}$, according to the manufacturer's protocol. JC-1 is a dye that is highly sensitive to the membrane potential (23). In healthy chondrocytes, $\Delta \psi \mathrm{m}$ was normal, and JC-1 was able to form polymers, exhibiting bright red fluorescence emission. By contrast, in apoptotic chondrocytes, $\Delta \psi \mathrm{m}$ decreased and JC-1 acquired a monomeric form, exhibiting green fluorescence emission. The $\Delta \psi \mathrm{m}$ was measured by flow cytometry within $30 \mathrm{~min}$ of staining. An excitation wavelength (Ex) at $488 \mathrm{~nm}$ and an emission wavelength (Em) at $530 \mathrm{~nm}$ were used to detect the JC-1 monomer. An Ex at $490 \mathrm{~nm}$ and an Em at $590 \mathrm{~nm}$ were used to detect the JC-1 polymer. Flow cytometry was performed using an INFLUX flow cytometer (BD Biosciences) and the data was analyzed using FlowJo (version 7.6.5, FlowJo LLC). 

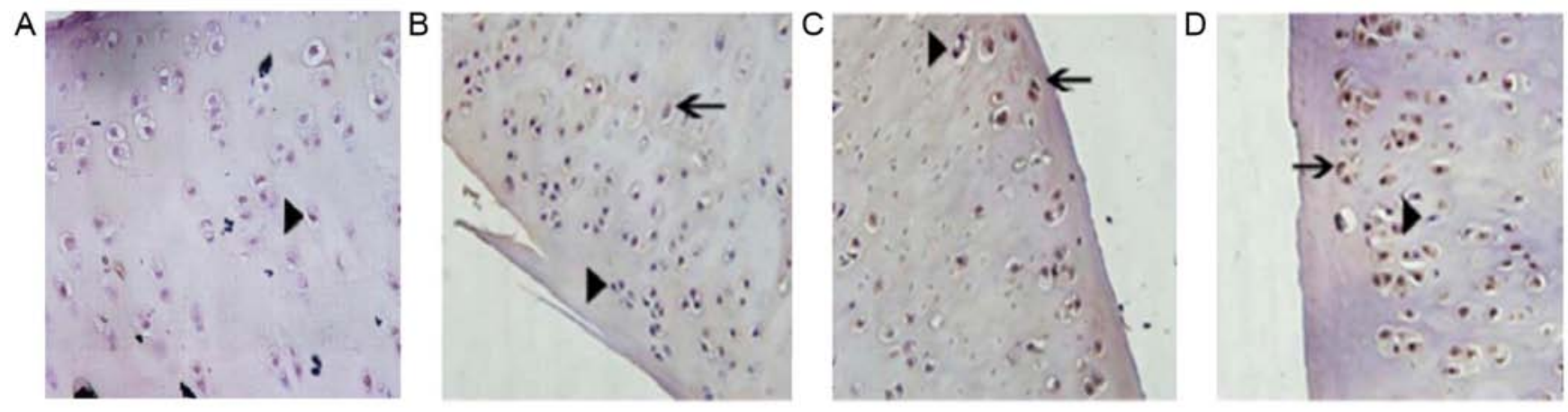

Figure 1. TUNEL results. (A) TUNEL staining of normal chondrocytes. The nuclei of normal cells were stained blue with hematoxylin and exhibited a normal shape. TUNEL staining of chondrocytes from patients with Outerbridge grades (B) I, (C) II and (D) III osteoarthritis. The nuclei of apoptotic cells were pyknotic and lost their normal shape. The chromatin was heterogeneously distributed and stained brown by hematoxylin. Increasing Outerbridge grades were associated with increasing numbers of apoptotic nuclei. Magnification, x100. Arrowheads indicate normal nuclei. Arrows indicate apoptotic nuclei.

TEM observation of chondrocytes. Chondrocytes were digested to prepare a monoplast suspension of $>1 \times 10^{5}$ cells $/ \mathrm{ml}$ and were treated according to the aforementioned procedures. The morphology of the chondrocytes was then observed by TEM as described.

Drug treatment. Monoplast suspensions $\left(1 \times 10^{5}\right.$ cells $\left./ \mathrm{ml}\right)$ of normal chondrocytes were divided into three groups: i) Normal control group, consisting of untreated chondrocytes; ii) Ro (Sigma-Aldrich; Merck KGaA) group, consisting of chondrocytes treated with $1.0 \mu \mathrm{mol} / 1$ Ro for $12 \mathrm{~h}$; and iii) Cur (Sigma-Aldrich; Merck $\mathrm{KGaA}$ ) + Ro group, consisting of chondrocytes treated with $2.0 \mu \mathrm{mol} / 1 \mathrm{Cur}$ for $4 \mathrm{~h}$ followed by treatment with $1.0 \mu \mathrm{mol} / \mathrm{l}$ Ro for $12 \mathrm{~h}$. All chondrocytes were cultured in an incubator with $5 \% \mathrm{CO}_{2}$ and saturated humidity at $37^{\circ} \mathrm{C}$.

Measurement of cell proliferation and apoptosis. The cultured primary chondrocytes were digested to prepare a monoplast suspension of $>1 \times 10^{4}$ cells $/ \mathrm{ml}$. Cellular proliferation was assessed using a Cell Counting Kit-8 (CCK-8; Applygen Technologies, Inc.). Optical density values of the three groups of cultured cells were measured and compared with the control group and Ro group, Ro group and Cur + Ro group (Independent-Samples T Test with subsequent Bonferroni correction, with $\mathrm{P}<0.025$ considered significant) every day. Chondrocytes were stained by Annexin V-EGFP and propidium iodide at $37^{\circ} \mathrm{C}$ for $15 \mathrm{~min}$, and cellular apoptosis was then assessed by flow cytometry (INFLUX flow cytometer; BD Biosciences) using an Annexin V-fluorescein isothiocyanate kit (Nanjing KeyGen Biotech Co., Ltd.), and the data was analyzed using FlowJo (version 7.6.5, FlowJo LLC). Moreover, the morphological features of apoptotic chondrocytes were observed via TEM as described.

$\Delta \psi m$ measurement of cultured chondrocytes. Normal and OA chondrocytes were harvested. Following drug treatment, mitochondria were extracted using a mitochondrial extraction kit (Nanjing KeyGen Biotech Co., Ltd.), according to the manufacturer's protocol. The $\Delta \psi \mathrm{m}$ was then measured by flow cytometry, as described.

Quantitative detection of type II collagen. Total protein was extracted from cultured and treated chondrocytes $\left(1 \times 10^{6}\right.$ cells $\left./ \mathrm{ml}\right)$ with the total protein extraction kit (Nanjing KeyGen Biotech Co., Ltd.), following the manufacturer's protocol, and the levels of type II collagen were assessed via a double antibody sandwich avidin-biotin complex-ELISA method [cat. no. (Col II) h143; Applygen Technologies, Inc.] (24). A standard curve was drawn in a semi-logarithmic coordinate paper by plotting the OD values from different concentrations of collagen II. Then, according to the OD value of the sample, the corresponding human collagen II content was found on the curve (24).

Statistical analysis. SPSS software (version 23.0; IBM Corp.) was used to perform statistical analyses. All data are reported as the mean \pm standard deviation. Comparisons between two groups were performed using a Student's t-test with subsequent Bonferroni correction, with $\mathrm{P}<0.025$ considered significant. Statistical differences among multiple groups were analyzed using one-way ANOVA followed by Bonferroni's post hoc test, with $\mathrm{P}<0.05$ considered significant.

\section{Results}

Detection of apoptotic cells in the cartilage tissue. The cartilage tissue was stained using a TUNEL assay. The nuclei of normal cells were stained blue and exhibited a normal shape (Fig. 1). Apoptotic chondrocytes were distributed unevenly in the articular cartilage; more apoptotic chondrocytes were identified in proximity to the tide line, and fewer apoptotic chondrocytes were present in the superficial and middle layers. The nuclei of apoptotic cells were pyknotic and lost their ellipse shape. The chromatin was condensed and the nuclei stained with the TUNEL assay were brown. The number of apoptotic cells in the cartilage tissue increased and the number of normal cells decreased with increasing Outerbridge grade (Fig. 1).

Chondrocytes in the superficial and transitional cell layers were selected for observation by TEM, as they are exposed to relatively normal oxygen pressure (25). The shape of the normal chondrocyte was oval, and the cell membrane forms an elliptical boundary (Fig. 2). Various organelles were visible in the cytoplasm, including a large number of mitochondria, the Golgi apparatus and the endoplasmic reticulum. The electron density of the outer mitochondrial membrane was high, even and continuous. The cristae were arranged in a regular pattern. 

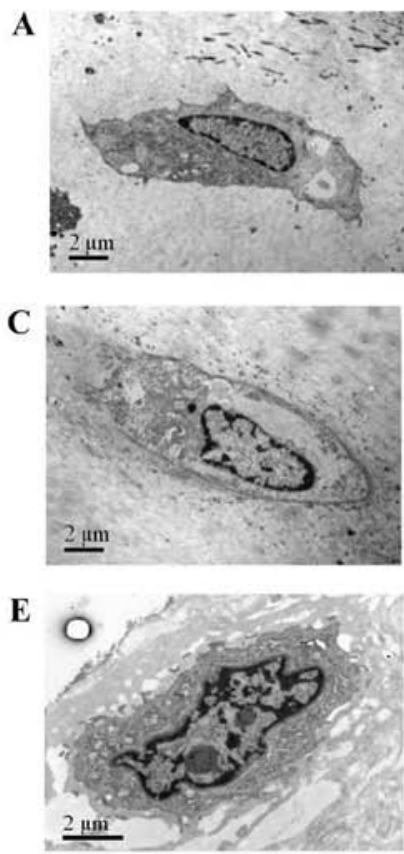
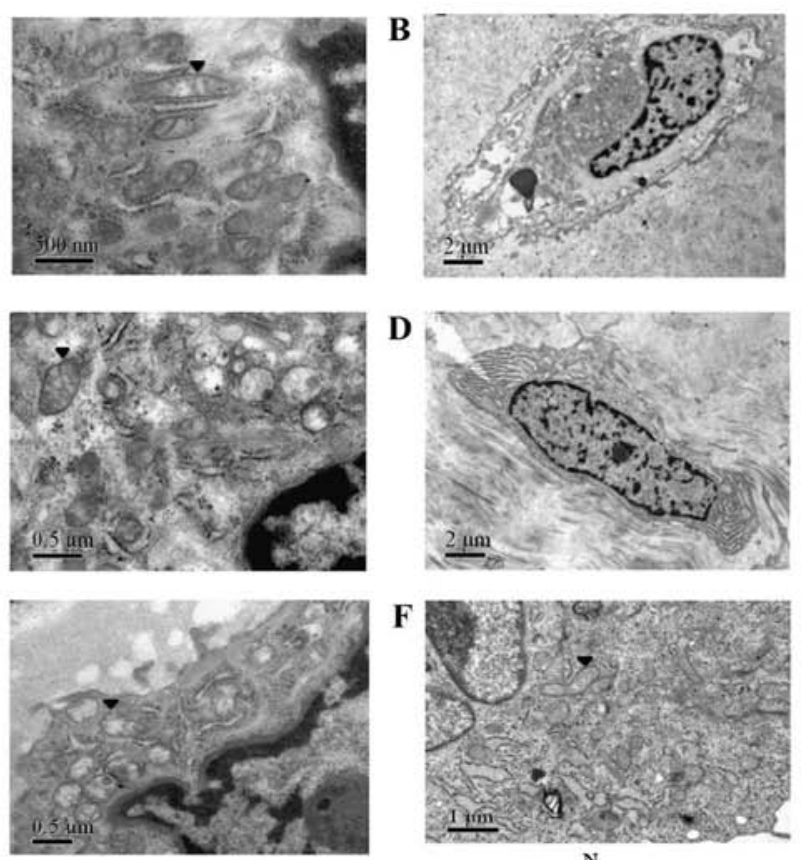

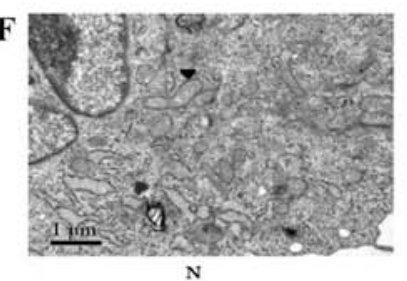

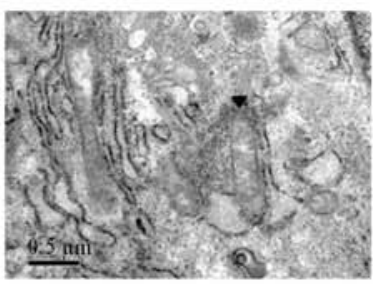
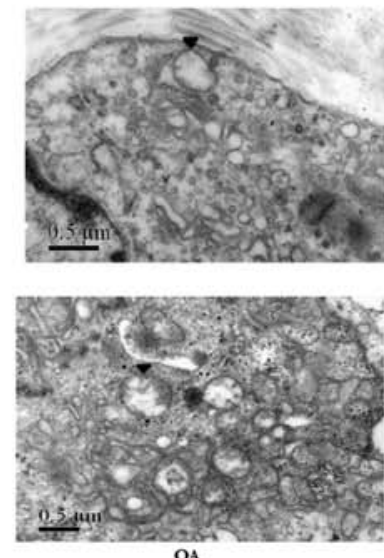

Figure 2. TEM results. (A) Normal chondrocyte. Normal chondrocytes were oval-shaped and the cell membrane formed an elliptical boundary. Various organelles were visible in the cytoplasm, including a large number of mitochondria, the Golgi apparatus and the endoplasmic reticulum. The electron density of the outer mitochondrial membrane was high, even and continuous. The cristae were organized in a regular pattern. TEM of transitional-layer OA chondrocytes from patients with Outerbridge grades (B) I, (C) II and (D) III was performed. Organelles in the chondrocytes decreased in number, and more mitochondria were swollen with increasing Outerbridge grade. Mitochondrial shape was altered from oblong to round. Cristae exhibited an irregular pattern and decreased in number. Electron density of the outer mitochondrial membrane became asymmetrical and discontinuous. (E) TEM of surface-layer OA chondrocytes. Chondrocytes had fewer cell processes and organelles in the cytoplasm. Mitochondria were swollen, the outer membrane electron density was uneven and the structure of the cristae was disrupted. (F) TEM of cultured chondrocytes. Compared with normal cells, the mitochondria of OA chondrocytes were swollen and abnormal, the electron density was uneven and discontinuous and the structure of the cristae was disrupted. Arrowheads indicate mitochondria. OA, osteoarthritis.

Moreover, morphological alterations in the mitochondria of OA chondrocytes in the transitional cell layer were observed. Chondrocytes from patients with Outerbridge grades I, II and III (Fig. 2B, C and D, respectively) exhibited a decreased number of organelles in the chondrocytes, and the mitochondria were more swollen with increasing Outerbridge grade. The mitochondrial shape was altered from oblong to round. Additionally, the regular pattern of the cristae was impaired and, in certain cases, cristae were not detected. The electron density of the outer mitochondrial membrane became asymmetrical and discontinuous.

The morphology of OA chondrocytes in the superficial layer was reminiscent of fibroblasts (Fig. 2E); however, OA chondrocytes exhibited fewer organelles in the cytoplasm compared with normal chondrocytes. Notably, the morphology of the mitochondria was similar to the transitional layer cells; the mitochondria were swollen, the outer membrane electron density was uneven, the structure of the cristae was impaired, and the number of cristae was decreased. The results of cultured chondrocytes suggested that, compared with normal cells, the mitochondria of OA chondrocytes were swollen and abnormal, the electron density was uneven and discontinuous, the cristae pattern was impaired and the number of cristae was reduced (Fig. 2F).

\section{Mitochondrial function in chondrocytes}

Detection of respiratory chain complex activity. To investigate whether OA impaired mitochondrial function in chondrocytes, quantification of the activities of the MRC complexes 1-4 and ATP synthase was performed. The results for these five enzymes in normal and OA chondrocytes showed constant variance $(\mathrm{P}=0.645,0.696,0.157$, 0.230 and 0.170 ). The activities of the MRC complexes 1,2 , $2+3$ and 4 and ATP synthase in normal chondrocytes were $207.77 \pm 38.26,161.60 \pm 22.66,288.38 \pm 17.30,218.80 \pm 29.63$ and $193.94 \pm 43.66 \mathrm{nmol} / \mathrm{min} / \mathrm{mg}$, respectively. The activities of the MRC complexes 1, 2, 2+3 and 4, and ATP synthase in OA chondrocytes were $180.18 \pm 34.80,141.68 \pm 22.10,261.54 \pm 27.01$, $194.52 \pm 20.29$ and $158.44 \pm 22.72 \mathrm{nmol} / \mathrm{min} / \mathrm{mg}$, respectively. The enzymatic activities in normal chondrocytes were significantly increased compared with OA chondrocytes (Table I).

$\Delta \psi m$ measurement of chondrocytes. JC-1, a carbocyclic lipophilic fluorescent dye, is a dye that is highly sensitive to the membrane potential (23). In chondrocytes collected from healthy tissues, $\Delta \psi \mathrm{m}$ was normal, and JC-1 was able to form polymers in the mitochondrial matrix, exhibiting bright red fluorescence emission (Fig. 3A). By contrast, in apoptotic chondrocytes, $\Delta \psi \mathrm{m}$ decreased and JC-1 acquired a monomeric form, exhibiting green fluorescence emission (Fig. 3B). During the late phases of apoptosis, the green fluorescence intensity increased (Fig. 3C). Compared with normal chondrocytes, the red fluorescence detected in OA chondrocytes was decreased, and the green fluorescence was increased. The ratio of red/green fluorescent signal in normal chondrocytes was $2.58 \pm 0.26$ (Fig. 4A), whereas the signal in OA chondrocytes was $1.50 \pm 0.35$ (Fig. 4B). Notably, the difference between OA and normal 
Table I. Activities of the mitochondrial respiratory chain enzyme complexes 1,2, 2+3 and 4, and ATP synthase.

\begin{tabular}{lccr}
\hline Enzyme complex & Normal, nmol/min/mg & Osteoarthritis, nmol/min/mg & P-value \\
\hline Complex 1 & $207.77 \pm 38.26$ & $180.18 \pm 34.80$ & $0.009^{\mathrm{a}}$ \\
Complex 2 & $161.60 \pm 22.66$ & $141.68 \pm 22.10$ & $0.027^{\mathrm{a}}$ \\
Complex 2+3 & $288.38 \pm 17.30$ & $261.54 \pm 27.01$ & $0.018^{\mathrm{a}}$ \\
Complex 4 & $218.80 \pm 29.63$ & $194.52 \pm 20.29$ & $0.034^{\mathrm{a}}$ \\
ATP synthase & $193.94 \pm 43.66$ & $158.44 \pm 22.72$ & $0.005^{\mathrm{a}}$ \\
\hline
\end{tabular}

${ }^{\mathrm{a}} \mathrm{P}<0.05$.
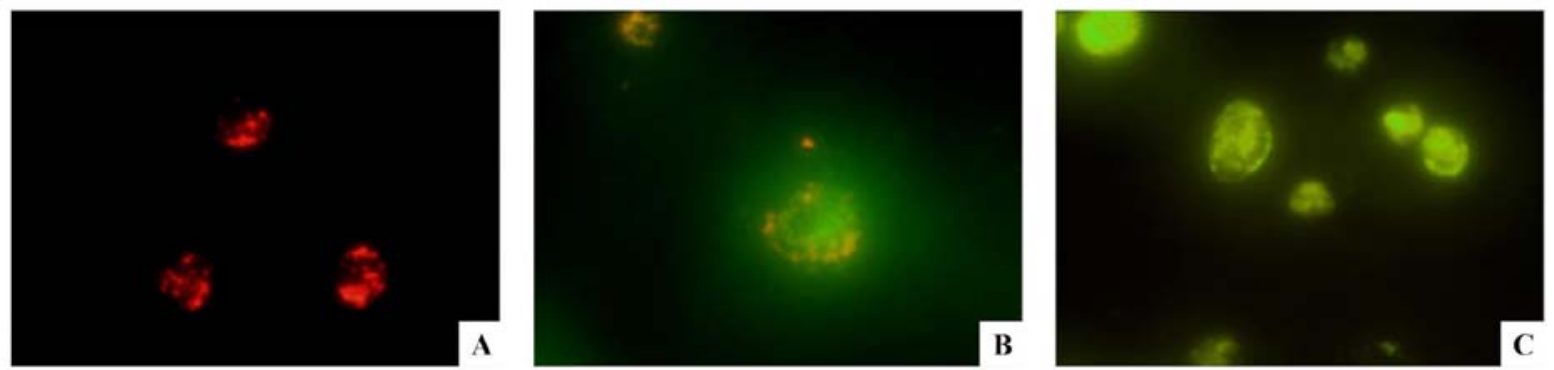

Figure 3. Fluorescence microscopy results. (A) In chondrocytes with normal $\Delta \psi \mathrm{m}$, JC-1 was able to form polymers in the mitochondrial matrix, exhibiting bright red fluorescence emission. (B) In apoptotic cells, $\Delta \psi \mathrm{m}$ decreased and JC-1 presented a monomeric structure, which exhibited green fluorescence. (C) In late-phase apoptosis, cells presented bright green fluorescence. Magnification, $x 1,000 . \Delta \psi \mathrm{m}$, mitochondrial membrane potential.

chondrocytes was statistically significant $(\mathrm{P}<0.01)$. The present result suggested that the mitochondrial membranes were depolarized in OA chondrocytes and that the mitochondrial function in OA chondrocytes was impaired. The present JC-1 results were consistent with the aforementioned TEM results.

\section{Drug treatment}

Cellular viability assay. To investigate the potential role of mitochondrial dysfunction in OA, a CCK-8 assay and Annexin-V staining were performed on chondrocytes cultured with Ro or Cur. The cell proliferation assay suggested that the proliferative ability of chondrocytes in the Ro group was significantly decreased compared with the control group from day 1 to day $5(\mathrm{P}<0.01)$. By contrast, the proliferative ability of chondrocytes in the Ro + Cur group significantly increased compared with the Ro group from day 1 to 5 . The protective effects of Cur increased in the slow growth and stationary phases (Fig. 5A). Moreover, the early, late and total apoptosis rates in the normal group were $0.58 \pm 0.14$, $3.80 \pm 0.26$ and $4.38 \pm 0.40 \%$, respectively (Fig. 5B). By contrast, the early, late and total apoptosis rates in the Ro group were $1.38 \pm 0.52,6.15 \pm 0.32$ and $7.53 \pm 0.84 \%$, respectively (Fig. 5C). Following Cur + Ro treatment, the apoptosis rates were $0.62 \pm 0.10$, $4.72 \pm 0.97$ and $5.34 \pm 1.07 \%$ (Fig. 5D). Notably, there was a significant difference between $\mathrm{OA}$ and Ro groups $(\mathrm{P}<0.01)$ and between Ro and Ro + Cur groups $(\mathrm{P}<0.01)$. The present results suggested that Ro increase the apoptosis rate in chondrocytes and that Cur was able to reduce the apoptosis rate induced by Ro.

TEM of cultured chondrocytes. The mitochondria in the Ro group were swollen and abnormal compared with the control group (Fig. 5E). The electron density of the outer membrane became heterogeneous, and the lumen of the cristae was expanded and collapsed (Fig. 5F). The mitochondrial morphology of the Cur + Ro group was not as severe as in the Ro group; however, it appeared affected compared with the control group, and a subset of mitochondria exhibited defects (Fig. 5G).

$\Delta \psi m$ measurement of cultured chondrocytes. The ratio of $\mathrm{red} /$ green fluorescence was $2.58 \pm 0.26$ in the control group, $1.78 \pm 0.24$ in the Ro group, and $2.23 \pm 0.14$ in the Cur + Ro group. Notably, there was a significant difference between control and Ro groups $(\mathrm{P}<0.01)$ and between Ro and Ro + Cur groups $(\mathrm{P}<0.01)$. The mitochondrial membranes were more depolarized in chondrocytes treated with Ro, whereas Cur reversed the defects in $\Delta \psi \mathrm{m}$ induced by Ro (Fig. $4 \mathrm{C}$ and D).

Quantitative detection of type II collagen. A standard curve was drawn using standard optical density (OD) values. The concentration of type II collagen was calculated based on the OD value detected. Compared with the Ro group $(44.6 \pm 7.1 \mu \mathrm{g} / \mathrm{l})$, the concentrations of collagen in the control $(72.9 \pm 24.3 \mu \mathrm{g} / \mathrm{l} ; \mathrm{P}=0.044)$ and Cur + Ro $(100.25 \pm 4.50 \mu \mathrm{g} / \mathrm{l}$; $\mathrm{P}<0.01)$ groups were significantly higher. Although the Cur + Ro group exhibited a higher collagen content than the control group, there was no significant difference $(\mathrm{P}=0.05)$. Additionally, the difference between the three groups was statistically significant (Table II).

\section{Discussion}

TEM imaging allows for the visualization of mitochondria. Notably, important mitochondrial morphological features are detectable using TEM. The outer membrane is a double-membrane structure with high permeability and the 

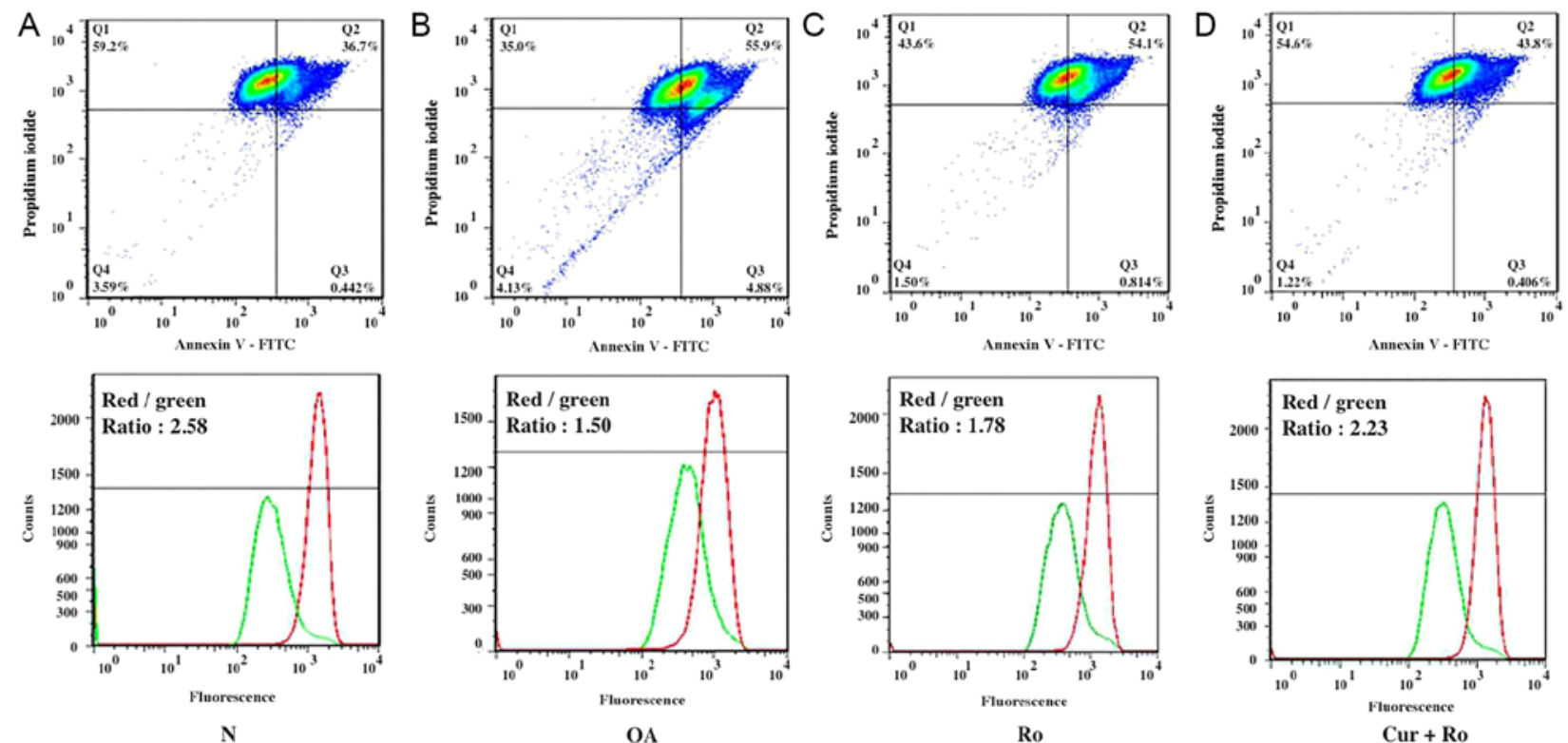

Figure 4. $\Delta \psi \mathrm{m}$ results. Compared with (A) normal chondrocytes, JC-1 staining suggested a decreased $\Delta \psi \mathrm{m}$ in (B) OA chondrocytes, which exhibited a lower $\mathrm{red} / \mathrm{green}$ fluorescence ratio. Red/green fluorescence ratios in the normal and OA groups were $2.58 \pm 0.26$ and $1.50 \pm 0.35$, respectively. (C) $\Delta \psi \mathrm{m}$ decreased in the Ro group, which presented a red/green fluorescence ratio of 1.78 \pm 0.24 . (D) $\Delta \psi \mathrm{m}$ in the Cur + Ro group was $2.23 \pm 0.15$, lower than that in the normal condition, but higher than that in the Ro group. There was a significant difference between control and Ro groups $(\mathrm{P}<0.01)$ and between Ro and Ro + Cur groups $(\mathrm{P}<0.01)$. Statistical differences among multiple groups were analyzed using one-way ANOVA followed by Bonferroni's post-hoc test. N, normal; OA, osteoarthritis; Ro, rotenone; Cur, curcumin; $\Delta \psi \mathrm{m}$, mitochondrial membrane potential; FITC, fluorescein isothiocyanate.

A

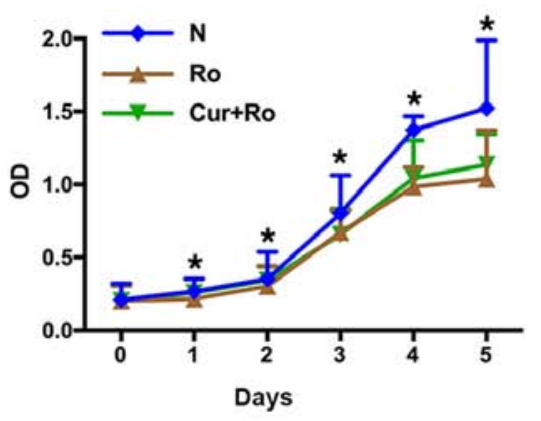

B

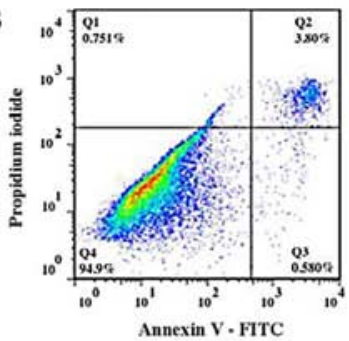

E

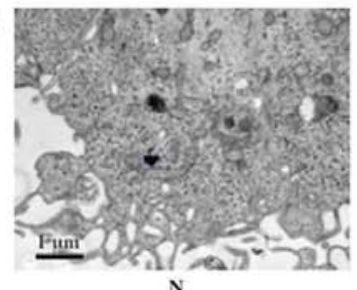

C

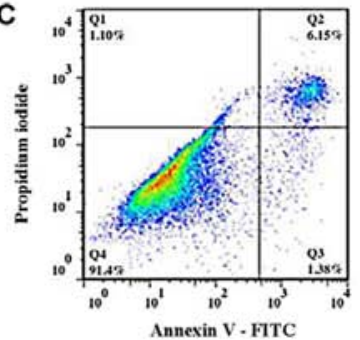

$\mathrm{F}$

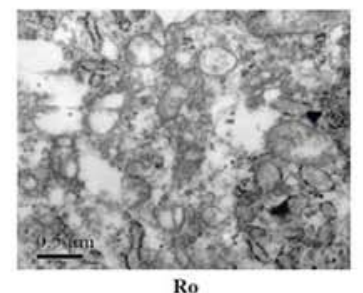

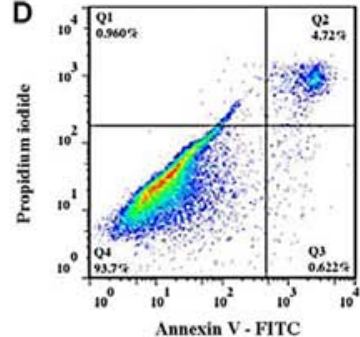

G

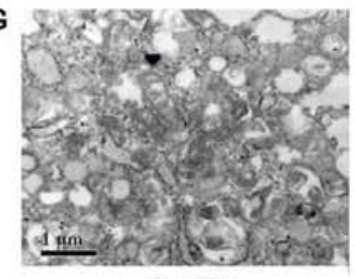

Figure 5. Drug treatment results. (A) The proliferative ability of chondrocytes in the Ro group was decreased compared with the normal group from day 1 to 5. There was a significant difference between control and Ro groups and between Ro and Ro + Cur groups. The proliferative ability of chondrocytes in the Ro + Cur group was increased compared with the Ro group. The effects of Cur appear to be more evident during the slow growth phase (0-2 days) and possibly during the stationary phase (4-5 days). (B) Early, late and total cellular apoptotic rates in the normal group were $0.58 \pm 0.14,3.80 \pm 0.26$ and $4.38 \pm 0.40 \%$, respectively. (C) Early, late and total cellular apoptotic rates in the Ro group were $1.38 \pm 0.52,6.15 \pm 0.32$ and $7.53 \pm 0.84 \%$, respectively. (D) Early, late and total cellular apoptotic rates in the Cur + Ro group were $0.62 \pm 0.11,4.72 \pm 0.97$ and $5.34 \pm 1.07 \%$, respectively. Compared with (E) normal chondrocytes, the mitochondria in the (F) Ro group were swollen and deformed. The electron density of the outer membrane became heterogeneous and the lumen of the cristae were expanded and collapsed. (G) Mitochondrial morphology of the Cur + Ro group was not as severe as in the Ro group; however, it appeared affected compared with the control group, and a subset of mitochondria exhibited defects. Arrowheads indicate mitochondria. " $\mathrm{P}<0.01$ between treatments. N, normal; Ro, rotenone; Cur, curcumin; OD, optical density; FITC, fluorescein isothiocyanate.

inner membrane contains the respiratory chain and enzymes for ATP synthesis, but is permeable only to ions and small molecules (10). Selective permeability is important to maintain the potential difference between the two sides of the membrane, and it is crucial to create the transmembrane proton gradient that is necessary for oxidative phosphorylation (10). The cristae are formed by folded inner membrane layers that increase the surface area of the inner membrane of the mitochondria. Notably, the morphology and number of the cristae are associated with cell metabolism. The more energy the cell consumes, the larger the total area of the mitochondrial inner membrane is expected to be, with mitochondria presenting a higher number of cristae (12). 
Table II. Collagen II content in chondrocytes.

\begin{tabular}{lc}
\hline Group & Collagen II, ng/ml \\
\hline Normal chondrocytes & $72.88 \pm 24.3$ \\
Rotenone & $44.63 \pm 7.11$ \\
Curcumin + Rotenone & $100.25 \pm 4.50$ \\
P-value & $0.007^{\mathrm{a}}$ \\
\hline
\end{tabular}

${ }^{\mathrm{a}} \mathrm{P}<0.05$

The present TEM images suggested that structural and morphological impairments of the mitochondria were increased with increasing Outerbridge grade in the transitional layer of the cartilage. In the early degenerative stage, various factors can cause the dysfunction of the MRC, including the opening of the mitochondrial permeability transition pore (PTP), the disruption of $\Delta \psi \mathrm{m}$ and the collapse of the outer membrane (26). Water molecules can enter the mitochondria through the PTP, causing the loss of normal mitochondrial morphology and mitochondrial swelling (26). Dysfunctional mitochondria exhibit swollen and wider cristae, which may collapse. Beregi and Regius (6) identified that, with aging, the mitochondria in human peripheral lymphocytes and skeletal muscle cells exhibit fewer cristae, and these are partially replaced by myeloid lamellar structures. These morphological changes are indicators of mitochondrial dysfunction. In fact, the number of morphological defects observed in mitochondria are directly associated with defects in the mitochondrial oxidative phosphorylation (27).

The present study suggested that the activities of the complexes of the MRC in OA chondrocytes were decreased compared with normal chondrocytes. Maneiro et al (11) examined the activity of the same complexes in patients with OA and found that $\mathrm{OA}$ chondrocytes exhibited decreased activity of MRC-complex II and III, which decreased by $35 \%$ and $13 \%$, respectively, and this decrease was not compensated by the increased activity of complex I. Lee et al (28) demonstrated that the number of mitochondria in OA chondrocytes was increased compared with normal chondrocytes, as measured by citrate synthase content. The results of Lee et al (28) suggested that the decrease in MRC complexes, the subsequent impairment of electron transfer and the insufficient generation of ATP may be compensated, at least in part, by an increase in the total number of mitochondria in the cartilage of patients with OA. Almeida et al (29) demonstrated that the activities of MRC complexes were decreased in OA chondrocytes compared with normal cells and that the decreased activity of MRC-complex I and II in OA chondrocytes was irreversible. This effect may be due to high concentrations of ROS or nitric oxide in the synovial fluid. Both transient and irreversible defects are able to severely affect mitochondrial function in the cartilage $(30,31)$. The present study suggested that the activities of the MRC complexes in abnormal OA chondrocytes were significantly decreased compared with normal cells.

$\Delta \psi \mathrm{m}$ plays an important role in maintaining mitochondrial membrane permeability and mitochondrial function. JC-1, a carbocyclic lipophilic fluorescent dye, is highly sensitive to the mitochondrial membrane potential (23). Although JC-1 presents certain limitations, such as poor sensitivity in case of a $\Delta \psi \mathrm{m}<100 \mathrm{mV}$, it is considered to be the gold standard for detecting $\Delta \psi \mathrm{m}$ (23). In the present study, $\Delta \psi \mathrm{m}$ was decreased in OA chondrocytes compared with normal cells, as suggested by the decreased ratio of red/green fluorescence following JC-1 staining. The present results suggested that the majority of mitochondria in OA chondrocytes were dysfunctional and $\Delta \psi \mathrm{m}$ was decreased. In addition, the inhibition of electron transport and oxidative phosphorylation, the opening of the PTP and the decrease in $\Delta \psi \mathrm{m}$ could directly cause mitochondrial swelling and collapse of the outer membrane (26). This phenotype was consistent with the present TEM results, suggesting that the mitochondrial dysfunction in OA chondrocytes was associated with defects in mitochondrial morphology.

MRC complex I is the first enzyme of the respiratory chain and it is the largest and most complex enzyme in the MRC (32). Therefore, complex I is very important in energy metabolism. Ro is a natural isoflavone produced by leguminous plants, and it is a specific inhibitor of the enzymatic activity of MRC complex I (14). Various previous studies used Ro to inhibit the respiratory chain $(15,16)$. In the present study, TEM results suggested that the mitochondria of chondrocytes treated with Ro exhibited morphological defects that were similar to the ones observed in mitochondria of chondrocytes isolated from Outerbridge grade III samples. $\Delta \psi \mathrm{m}$ was decreased in the Ro group, as detected using the JC-1 method. Additionally, cell proliferation was decreased, the apoptotic rate was increased and the expression of type II collagen was significantly decreased. The present results suggested that inhibition of the MRC and reduction of the mitochondrial function following Ro treatment could induce pathological changes in chondrocytes similar to those observed in OA. In the present study, these pathological changes were found to be associated with metabolic dysfunction, increased ROS and impaired $\Delta \psi \mathrm{m}$.

Furthermore, Cur was used in the present study to attenuate the effects of Ro. After pre-treatment with Cur for $4 \mathrm{~h}$, the chondrocytes were protected against Ro-induced mitochondrial dysfunction. The ratio of red/green fluorescence intensity increased from 1.78 to 2.23 , cell proliferation increased and the apoptotic rate was reduced from $9.01 \%$ to $4.44 \%$, a similar level that that of the normal group (3.98\%). The ability of chondrocytes to secrete type II collagen in the Cur + Ro group was increased compared with the Ro group, and it was similar to the control group. Reddy and Lokesh (33) demonstrated that the antioxidant effects of Cur were mediated by a stabilization of antioxidant enzymes, such as superoxide dismutase (SOD), catalase and glutathione peroxidase, and by a reduction in lipid peroxidation. Koiram et al (34) showed that Cur had protective effects on the activity of SOD and other antioxidant enzymes from radiation, leading to reduced cell damage. Therefore, the antioxidant effects of Cur could neutralize the ROS produced by mitochondria following Ro-mediated MRC enzyme I inhibition, preventing the mitochondrial damage caused by oxidative stress, stabilizing the mitochondrial membrane, protecting mitochondrial function, increasing the secretion of type II collagen and reducing the apoptotic rate of chondrocytes. 
The present study had some limitations. For example, all factors affecting the metabolism of chondrocytes have effects on both the superficial and deep layers of the cartilage. Previous studies demonstrated that oxygen and nutrients diffusing through the synovial fluid may have an important role in chondrocytes in the deeper cartilage layers $(2,25)$. However, in the present study, chondrocytes from different layers were not examined. Notably, in the cartilage, aerobic respiration and anaerobic glycolysis occur in parallel, also under aerobic conditions (3). Therefore, lactic acid and pyruvic acid generated by anaerobic glycolysis may act as free radical scavengers, increasing the synthesis of ATP despite a decreased enzymatic activity of the MRC $(35,36)$. Additionally, adequate oxygen and glucose supply may partially restore the function of chondrocyte mitochondria, thus affecting the experimental results. Moreover, the chondrocytes were cultured under standard oxygen conditions. Therefore, the morphology and function of chondrocytes in vitro may not reflect the physiological in vivo state.

\section{Acknowledgements}

Not applicable.

\section{Funding}

No funding was received.

\section{Availability of data and materials}

The datasets used and/or analyzed during the current study are available from the corresponding author on reasonable request.

\section{Authors' contributions}

ZL, HL and YoC designed the experiments. ZL, HL, YuC, XY, $\mathrm{ZM}$ and RW performed the experiments and analyzed the data. ZL, HL and ZM wrote the manuscript and revised the manuscript. All authors read and approved the final manuscript.

\section{Ethics approval and consent to participate}

The present study was approved by The Ethics Committee of Peking University First Hospital, and all participants provided informed consent [approval no. 2012 (532)].

\section{Patient consent for publication}

Not applicable.

\section{Competing interests}

The authors declare that they have no competing interests.

\section{References}

1. Blanco FJ, Guitian R, Vázquez-Martul E, de Toro FJ and Galdo F: Osteoarthritis chondrocytes die by apoptosis: A possible pathway for osteoarthritis pathology. Arthritis Rheum 41: 284, 1998.

2. Buckwalter JA and Mankin HJ: Articular cartilage: Degeneration and osteoarthritis, repair, regeneration, and transplantation. Instr Course Lect 47: 487-504, 1998.
3. Otte P: Basic cell metabolism of articular cartilage. Manometric studies. Z Rheumatol 50: 304-312, 1991.

4. Falchuk KH, Goetzl EJ and Kulka JP: Respiratory gases of synovial fluids. An approach to synovial tissue circulatory-metabolic imbalance in rheumatoid arthritis. Am J Med 49: 223-231, 1970.

5. Morgan-Hughes JA, Darveniza P, Kahn SN, Landon DN, Sherratt RM, Land JM and Clark JB: A mitochondrial myopathy characterized by a deficiency in reducible cytochrome b. Brain 100: 617-640, 1977.

6. Beregi E and Regius O: Comparative morphological study of age related mitochondrial changes of the lymphocytes and skeletal muscle cells. Acta Morphol Hung 35: 219-224, 1987.

7. Takamiya S, Yanamura W, Capaldi RA, Kennaway NG, Bart R, Sengers RC, Trijbels JM and Ruitenbeek W: Mitochondrial myopathies involving the respiratory chain: A biochemical analysis. Ann N Y Acad Sci 488: 33-43, 1986.

8. Ishikawa Y, Chin JE, Hubbard HL and Wuthier RE: Utilization and formation of amino acids by chicken epiphyseal chondrocytes: Comparative studies with cultured cells and native cartilage tissue. J Cell Physiol 123: 79-88, 1985.

9. Kühn K, D'Lima DD, Hashimoto S and Lotz M: Cell death in cartilage. Osteoarthritis Cartilage 12: 1-16, 2004.

10. Perkins G, Renken C, Martone ME, Young SJ, Ellisman M and Frey T: Electron tomography of neuronal mitochondria: Three-dimensional structure and organization of cristae and membrane contacts. J Struct Biol 119: 260-272, 1997.

11. Maneiro E, Martín MA, de Andres MC, López-Armada MJ, Fernández-Sueiro JL, del Hoyo P, Galdo F, Arenas J and Blanco FJ: Mitochondrial respiratory activity is altered in osteoarthritic human articular chondrocytes. Arthritis Rheum 48: 700-708, 2003.

12. Ernster L and Schatz G: Mitochondria: A historical review. J Cell Biol 91: 227s-255s, 1981.

13. Chauvin C, De Oliveira F, Ronot X, Mousseau M, Leverve X and Fontaine E: Rotenone inhibits the mitochondrial permeability transition-induced cell death in U937 and KB cells. J Biol Chem 276: 41394-41398, 2001.

14. Navarro A, Bández MJ, Gómez C, Repetto MG and Boveris A: Effects of rotenone and pyridaben on complex I electron transfer and on mitochondrial nitric oxide synthase functional activity. J Bioenerg Biomembr 42: 405-412, 2010.

15. DiDonato S, Zeviani M, Giovannini P, Savarese N, Rimoldi M, Mariotti C, Girotti F and Caraceni T: Respiratory chain and mitochondrial DNA in muscle and brain in Parkinson's disease patients. Neurology 43: 2262-2268, 1993.

16. Li N, Ragheb K, Lawler G, Sturgis J, Rajwa B, Melendez JA and Robinson JP: Mitochondrial complex I inhibitor rotenone induces apoptosis through enhancing mitochondrial reactive oxygen species production. J Biol Chem 278: 8516-8525, 2003.

17. Jackson JK, Higo T, Hunter WL and Burt HM: The antioxidants curcumin and quercetin inhibit inflammatory processes associated with arthritis. Inflamm Res 55: 168-175, 2006.

18. Cole GM, Teter B and Frautschy SA: Neuroprotective effects of curcumin. Adv Exp Med Biol 595: 197-212, 2007.

19. Asai A and Miyazawa T: Dietary curcuminoids prevent high-fat diet-induced lipid accumulation in rat liver and epididymal adipose tissue. J Nutr 131: 2932-2935, 2001.

20. Outerbridge RE: The etiology of chondromalacia patellae. J Bone Joint Surg Br 43: 752-757, 1961.

21. Klagsbrun M: Large-scale preparation of chondrocytes. Methods Enzymol 58: 560-564, 1979.

22. Morgan-Hughes JA, Hayes DJ, Clark JB, Landon DN, Swash M, Stark RJ and Rudge P: Mitochondrial encephalomyopathies: Biochemical studies in two cases revealing defects in the respiratory chain. Brain 105: 553-582, 1982.

23. Shapiro HM: Membrane potential estimation by flow cytometry. Methods 21: 271-279, 2000.

24. Kongtawelert P and Ghosh P: A new sandwich-ELISA method for the determination of keratan sulphate peptides in biological fluids employing a monoclonal antibody and labelled avidin biotin technique. Clin Chim Acta 195: 17-26, 1990.

25. Aigner T, Kurz B, Fukui N and Sandell L: Roles of chondrocytes in the pathogenesis of osteoarthritis. Curr Opin Rheumatol 14: $578-584,2002$.

26. Ma Q, Fang H, Shang W, Liu L, Xu Z, Ye T, Wang X, Zheng M, Chen $Q$ and Cheng H: Superoxide flashes: Early mitochondrial signals for oxidative stress-induced apoptosis. J Biol Chem 286: 27573-27581, 2011

27. Higuchi M, Proske RJ and Yeh ET: Inhibition of mitochondrial respiratory chain complex I by TNF results in cytochrome c release, membrane permeability transition, and apoptosis. Oncogene 17: 2515-2524, 1998 
28. Lee HC, Yin PH, Lu CY, Chi CW and Wei YH: Increase of mitochondria and mitochondrial DNA in response to oxidative stress in human cells. Biochem J 348: 425-432, 2000.

29. Almeida A, Almeida J, Bolaños JP and Moncada S: Different responses of astrocytes and neurons to nitric oxide: The role of glycolytically generated ATP in astrocyte protection. Proc Natl Acad Sci USA 98: 15294-15299, 2001.

30. Goossens V, Stangé G, Moens K, Pipeleers D and Grooten J: Regulation of tumor necrosis factor-induced, mitochondria- and reactive oxygen species-dependent cell death by the electron flux through the electron transport chain complex I. Antioxid Redox Signal 1: 285-295, 1999.

31. Yook YH, Kang KH, Maeng O, Kim TR, Lee JO, Kang KI, Kim YS, Paik SG and Lee H: Nitric oxide induces BNIP3 expression that causes cell death in macrophages. Biochem Biophys Res Commun 321: 298-305, 2004.

32. Distelmaier F, Koopman WJ, van den Heuvel LP, Rodenburg RJ, Mayatepek E, Willems PH and Smeitink JA: Mitochondrial complex I deficiency: From organelle dysfunction to clinical disease. Brain 132: 833-842, 2009.

33. Reddy AC and Lokesh BR: Studies on spice principles as antioxidants in the inhibition of lipid peroxidation of rat liver microsomes. Mol Cell Biochem 111: 117-124, 1992.
34. Koiram PR, Veerapur VP, Kunwar A, Mishra B, Barik A, Priyadarsini IK and Mazhuvancherry UK: Effect of curcumin and curcumin copper complex (1:1) on radiation-induced changes of anti-oxidant enzymes levels in the livers of Swiss albino mice. J Radiat Res (Tokyo) 48: 241-245, 2007.

35. Le Goffe C, Vallette G, Jarry A, Bou-Hanna C and Laboisse CL: The in vitro manipulation of carbohydrate metabolism: A new strategy for deciphering the cellular defence mechanisms against nitric oxide attack. Biochem J 3: 643-648, 1999.

36. Aulwurm UR and Brand KA: Increased formation of reactive oxygen species due to glucose depletion in primary cultures of rat thymocytes inhibits proliferation. Eur J Biochem 267: 5693-5698, 2000.

This work is licensed under a Creative Commons Attribution-NonCommercial-NoDerivatives 4.0 International (CC BY-NC-ND 4.0) License. 